\title{
Gesund älter werden - ein nationales Gesundheitsziel
}

RUDOLF HERWECK UND

MARTINA THELEN

Rudolf Herweck, Vorsitzender der AG »Gesund älter werden «, Kooperationsverbund gesundheitsziele.de

Martina Thelen, Projektkoordinatorin Geschäftsstelle gesundheitsziele.de, c/o GVG e. V., Köln

Der Kooperationsverbund gesundheitsziele.de hat ein neues nationales Gesundheitsziel für ältere Menschen erarbeitet. Das Alter ist ein eigener Lebensabschnitt. Es gilt, für diese Lebensphase im Sinne einer Prävention im Alter Verbesserungen zu erreichen. Möglichkeiten der Einflussnahme auf die Gesundheit im Alter sind heutzutage sehr vielfältig. Bei dem Gesundheitsziel "Gesund älter werden" geht es somit nicht nur um Kuration im engen Sinn, sondern vor allem auch um Gesundheitsförderung und Prävention in Bezug auf gesundheitliche Ressourcen, Bildung, Wohnen und Wohnumgebung sowie Zugang zur gesundheitlichen Versorgung. Mit insgesamt 13 themenspezifischen Zielformulierungen und dazugehörigen Empfehlungen für Maßnahmen richtet sich das Gesundheitsziel an die Akteure und Umsetzer innerhalb und außerhalb unseres Gesundheitswesens. Es gilt, den aktuellen und zukünftigen altersbedingten Herausforderungen und Chancen zielorientiert zu begegnen - das nationale Gesundheitsziel "Gesund älter werden " bietet ferner die Aussicht, zahlreiche Akteure einzubinden, ihre Kräfte zu bündeln und so die Wirkung ihrer Maßnahmen zu verstärken.

\section{Einleitung}

Gesundheitsziele sind ein Steuerungsinstrument der Gesundheitspolitik. Mit ihnen soll das Gesundheitswesen stärker auf konkrete Ziele ausgerichtet werden und so zur Verbesserung der Gesundheit der Bevölkerung beitragen.

Gesundheitsziele können auf internationaler, nationaler und kommunaler Ebene definiert werden. Bereits 1981 verabschiedete die Weltgesundheitsorganisation (WHO) das Programm Health for All by the Year 2000 (HFA 2000), zu dem die Mitgliedsstaaten der Europäischen Region der WHO eine eu- ropäische Strategie zur Zielerreichung entwickelten.

Der Sachverständigenrat zur Begutachtung der Entwicklung im Gesundheitswesen hat in seinen Gutachten wiederholt ein Defizit an gesundheitspolitischer Orientierung festgestellt und deshalb die Entwicklung von Gesundheitszielen gefordert. ${ }^{1}$

In Deutschland mit seinem pluralistischen und stark gegliederten Gesundheitswesen ist ein die verschiedenen Akteure innerhalb und außerhalb des Gesundheitswesens zusammenführender Gesundheitszieleprozess besonders wichtig. Er bietet den Akteuren die 
Möglichkeit, sich auf eine gemeinsame Zielorientierung zu verständigen, gemeinsam besondere Anstrengungen zu unternehmen, um vorhandene Ungleich-

\section{In Deutschland ist die Plattform für die Entwicklung nationaler Gesundheitsziele der Kooperationsverbund gesundheitsziele.de}

heiten $\mathrm{zu}$ beseitigen und bestimmte schwer erreichbare Bevölkerungsgruppen zu erreichen und darüber hinaus weitere Akteure einzubeziehen, die auf die Gesundheit der Bevölkerung einwirken können (wie z. B. Kommunen, Patient(inn)enorganisationen, Selbsthilfeorganisationen, sonstige Vereinigungen und Verbände).

In Deutschland ist die Plattform für die Entwicklung nationaler Gesundheitsziele der Kooperationsverbund gesundheitsziele.de, ein Zusammenschluss der relevanten Akteure im deutschen Gesundheitswesen. Seit 2000 arbeiten Vertreterinnen und Vertreter der Kostenträger, der Leistungserbringer, der Sozialpartner, des Bundes, der Länder und der Kommunen, der Selbsthilfe- und Patient(inn)enorganisationen sowie der Wissenschaft und der Wirtschaft in diesem Kooperationsverbund zusammen. Sie entwickeln im Konsens und auf der Grundlage evidenzbasierter Erkenntnisse Gesundheitsziele, empfehlen Maßnahmen zur Zielerreichung und stoßen Selbstverpflichtungen der verantwortlichen Akteure an.

Bisher hat gesundheitsziele. de die folgenden Gesundheitsziele entwickelt und arbeitet an ihrer Umsetzung:

- Diabetes mellitus Typ 2: Erkrankungsrisiko senken, Erkrankte früh erkennen und behandeln

- Brustkrebs: Mortalität vermindern, Lebensqualität erhöhen

- Tabakkonsum reduzieren

- Gesund aufwachsen: Lebenskompetenz, Bewegung, Ernährung

- Gesundheitliche Kompetenz erhöhen, Patient(inn)ensouveränität stärken

- Depressive Erkrankungen: verhindern, früh erkennen, nachhaltig behandeln

Hinzu gekommen ist das kürzlich entwi- ckelte siebte Gesundheitsziel: »Gesund älter werden«, das von einer 31-köpfigen interdisziplinären Arbeitsgruppe $(\mathrm{AG})^{2}$ des Kooperationsverbundes gesundheitsziele.de erarbeitet wurde. Aufgabe der AG war es, in Bezug auf ein gesundes Altern älterer Menschen Ziele $\mathrm{zu}$ bestimmen, die in Zukunft erreicht werden sollen, und hierfür exemplarisch Maßnahmen vorzuschlagen, die zur Erreichung der Ziele hinführen.

\section{Ausgangspunkte}

Eine wichtige Konsequenz der demografischen Entwicklung für die Gesundheitsversorgung ist die Alterung der Bevölkerung mit dem relativen und absoluten Anstieg der Zahl älterer Menschen und insbesondere dem Anstieg der Zahl hochaltriger Menschen. Dieser Anstieg hat erhebliche Auswirkungen auf das Krankheitsgeschehen, sowohl in Bezug auf die Häufigkeit als auch was das Spektrum der Krankheiten anbelangt. Denn mit zunehmendem Alter, besonders deutlich im höheren und

1 Sachverständigenrat zur Begutachtung der Entwicklung im Gesundheitswesen (SVR). Gutachten 2005. Koordination und Qualität im Gesundheitswesen. Stuttgart: Kohlhammer Verlag; 2006. Sachverständigenrat zur Begutachtung der Entwicklung im Gesundheitswesen (SVR): Koordination und Integration - Gesundheitsversorgung in einer Gesellschaft des längeren Lebens. Bundestags-Drucksache 16/13770. Berlin: Deutscher Bundestag; 2009.

2 Mitglieder der Arbeitsgruppe: Rudolf Herweck, Bundesarbeitsgemeinschaft der Senioren-Organisationen e. V. (BAGSO), Bonn (Vorsitz), Rebecka Andyick, Deutsches Zentrum für Altersfragen, Berlin, Dr. Stefan Bluiher, Institut für Medizinische Soziologie, Berlin, Dr. Andreas Böhm, Ministerium für Umwelt, Gesundheit und Verbraucherschutz des Landes Brandenburg, Potsdam, Dr. Thomas Brockow, Deutsches Institut für Gesundheitsforschung gGmbH, Bad Elster, Karin Brösicke, Bundesärztekammer, Berlin, Erwin Dehlinger, AOK-BV, Berlin, Uwe Dresel, DAK, Hamburg, Wiebke Flor, Bundesministerium für Gesundheit, Bonn, Gunnar Geuter, Landeszentrum Gesundheit Nordrhein-Westfalen, Bielefeld, Dr. Hans Peter Huber, Deutsche Gesellschaft für AlterszahnMedizin e. V. (DGAZ), Berlin, Dr. Monika Köster, Bundeszentrale für gesundheitliche Aufklärung, Köln, Prof. Dr. Adelheid Kuhlmey, Institut für Medizinische Soziologie, Berlin, Dr. Susanne Kümpers, Wissenschaftszentrum Berlin (WZB), Karin Niederbühl, Verband der Ersatzkassen e. V. (vdek), Berlin, Lissy Nitsche-Neumann, Deutsches Institut für Gesundheitsforschung gGmbH, Bad Elster, Dr. Horst Peretzki, Bundesministerium für Gesundheit, Bonn, Dr. Stephan höchsten Alter, steigt die Wahrscheinlichkeit des Auftretens von Krankheiten und von funktionellen Einschränkungen an. Festzustellen ist auch eine Zunahme von chronischen Erkrankungen und von Multimorbidität. ${ }^{3}$ Im Vordergrund des somatischen Krankheitsgeschehens bei älteren Männern und Frauen stehen Herz-Kreislauf-Erkrankungen und Krankheiten des Bewegungsapparats. Von großer Bedeutung sind auch Krebserkrankungen. ${ }^{4}$ Anders als bei den somatischen Erkrankungen scheint der Anteil psychisch Kranker unter den älteren Menschen ähnlich hoch zu sein wie im jungen und mittleren Erwachsenenalter. Im Vordergrund stehen bei älteren Menschen allerdings demenzielle Erkrankungen ${ }^{5}$ sowie Depressionen. ${ }^{6}$ Viele ältere Menschen leiden darüber hinaus unter Schmerzen und Störungen der Körperfunktionen, wie zum Beispiel Bewegungseinschränkungen, Schlafstörungen, Harninkontinenz oder dem Nachlassen von Sinnesorganen oder Gedächtnis.?

Allgemein gilt, dass ein Teil des Krankheitsgeschehens im Alter beeinflussbar ist. Viele der Krankheiten kön-

Pitum-Weber, IKK e. V., Berlin, Dr. Beate Grossmann, Bundesvereinigung Prävention und Gesundheitsförderung e. V. (BVPG), Bonn, Dr. Anke-Christine Saß, Robert Koch-Institut (RKI), Berlin, Prof. Dr. Doris Schaeffer, Institut für Pflegewissenschaften, Universität Bielefeld, Dr. Christa Scheidt-Nave, Robert Koch-Institut (RKI), Berlin, Prof. Dr. Heiko Schneitler, Deutscher Städtetag, Köln (bis Juni 2011), Andreas Schumann, Sächsisches Staatsministerium für Soziales und Verbraucherschutz, Dresden, Prof. Dr. Clemens Tesch-Römer, Deutsches Zentrum für Altersfragen, Deutsche Gesellschaft für Gerontologie und Geriatrie e. V., Berlin, Martina Thelen, Gesellschaft für Versicherungswissenschaft und -gestaltung e. V. (GVG), Köln, Prof. Dr. Ulla Walter, Medizinische Hochschule Hannover, Petra Weritz-Hanf, Bundesministerium für Familie, Senioren, Frauen und Jugend, Berlin, Dr. Susanne Wurm, Deutsches Zentrum für Altersfragen, Berlin, Prof. Dr. Susanne Zank, Bundespsychotherapeutenkammer, Berlin, Dr. Jochen P. Ziegelmann, Deutsches Zentrum für Altersfragen, Berlin

3 Kohler M, Ziese T. Telefonischer Gesundheitssurvey des Robert Koch-Instituts zu chronischen Krankheiten und ihren Bedingungen. Berlin: Robert Koch-Institut; 2004.

4 Robert Koch-Institut. Schätzung der Krebsneuerkrankungen in Deutschland durch das Zentrum für Krebsregisterdaten (ZfKD) [Internet]. Datenbankabfrage 2011. verfügbar unter: http://www.rki.de/cln_169/ nn_204124/DE/Content/GBE/DachdokKrebs/Datenbankabfragen/datenbankabfragen node.html? nnn=true [zitiert am 08.04.2011] 
nen durch Gesundheitsförderung und Prävention vermieden, ihr Eintritt kann hinausgeschoben oder ihre Folgen können verringert werden. Maßnahmen der Gesundheitsförderung und der Prävention machen also bis in das hohe Alter Sinn. Aus diesem Grund muss besonderer Wert auf Gesundheitsförderung und Prävention gelegt werden. Von besonderer Bedeutung für die Gesundheit (auch) älterer Menschen ist deren soziale Lage. Ungünstige Umwelt- oder Lebensverhältnisse können die Gesundheit älterer Menschen negativ beeinflussen. Maßnahmen der Gesundheitsförderung und der Prävention sollten sich nicht nur auf die gesamte Altersgruppe, sondern auch auf spezifische Risikogruppen beziehen. Gerade um die Gesundheitschancen Letzterer zu verbessern, bedarf es besonderer Zugangswege und Maßnahmen.

Auch unzutreffende Altersbilder können Einfluss auf die Gesundheit älterer Menschen haben. Sie können sowohl Betroffenen als auch Professionellen die notwendige Unterscheidung zwischen dem natürlichen Altersprozess und krankheitsbedingten Entwicklungen

\section{Unzutreffende Altersbilder können Einfluss auf die Gesundheit älterer Menschen haben.}

stimmt. Wesentlich ist darüber hinaus auch eine Reihe von Umweltfaktoren, Lebensumständen und Verhaltensweisen, die zugunsten von Lebensqualität und Gesundheit beeinflussbar sind. Die AG hat deshalb im Rahmen von Gesundheitsförderung und Prävention das Erreichen folgender 5 Ziele für besonders wichtig gehalten und sich damit beschäftigt, wie die Rahmenbedingungen beschaffen sein sollten, damit diese Ziele erreicht werden können.

\section{Ziel 1: Die gesellschaftliche Teilha- be älterer Menschen ist gestärkt. Mangelnde Teilhabe und Isolation werden erkannt und gemindert.}

Der Begriff der gesellschaftlichen Teilhabe kann viele Bereiche umfassen und reicht von einer Mitgliedschaft in Vereinen, Verbänden oder informellen Gruppen über ehrenamtliches Engagement und politische Partizipation bis hin zu weiteren (Freizeit-) Tätigkeiten wie z. B. Lesen, Hausarbeit, Einkaufen oder körperliche Aktivität. Untersuchungen belegen, dass gesellschaftliche Teilhabe zur Gesunderhaltung und zur Lebensqualität beiträgt. ${ }^{8}$ Gesellschaftliche Teilhabe geschieht vielfach nicht voraussetzungslos. Damit ältere Menschen gesellschaftliche Teilhabe leben können, wird eine Reihe von Maßnahmen vorgeschlagen, die Menschen dabei unterstüterschweren. Wer als Betroffener oder als Arzt eine Erkrankung als altersbedingt ansieht, wird auch keine Schritte zur Heilung unternehmen. Wichtig sind deshalb realistische Altersbilder in der Bevölkerung und insbesondere bei den Professionellen, aber auch bei den älteren Menschen selbst. Besondere Bedeutung hat dies für die Verbesserung der Situation bei Demenzerkrankung und bei psychischen Erkrankungen.

\section{3 "Gesund älter werden" - Handlungsfelder, Ziele und Maßnahmen}

\subsection{Gesundheitsförderung und Prävention}

Die Lebensqualität und die Gesundheit älterer Menschen werden nicht nur von der gesundheitlichen Versorgung be- zen, gesellschaftliche Teilhabe (weiter) auszuüben. Sie reichen von der Schaffung sog. Ermöglichungs-, Gelegenheitsund Unterstützungsstrukturen bis hin zu konkreten Angeboten oder Unterstützung im Einzelfall. Zusätzlich werden Maßnahmen vorgeschlagen, die darauf hinwirken, Barrieren für Teilhabe und freiwilliges Engagement abzubauen.

\section{Ziel 2: Gesundheitliche Res-} sourcen und die Widerstandskraft älterer Menschen sind gestärkt und ihre gesundheitliche Risiken sind gemindert.

Vorhandene gesundheitliche Ressourcen und Widerstandskraft können die Gesundheit ebenso positiv beeinflussen, wie sie gesundheitliche Risiken negativ beeinflussen können.

Mit Rücksicht auf die Bedeutung der Bildung sowie des Wissens um die positive Veränderbarkeit des eigenen Älterwerdens für die gesundheitlichen Ressourcen älterer Menschen werden Maßnahmen zur Förderung des lebenslangen Lernens vorgeschlagen. Auch werden Maßnahmen zur Vermittlung eines positiveren Altersbildes und von mehr Selbstbewusstsein in Bezug auf die Möglichkeit der positiven Beeinflussung der eigenen Gesundheit empfohlen. Zur Vermeidung gesundheitlicher Risiken und zur Förderung gesundheitsförderlicher Aktivitäten wird dargestellt, wie gesundheitliche Kontrollmöglichkeiten gestärkt werden können. Ferner werden in Bezug auf Risiken für ältere Menschen Lösungsansätze zur besseren Erkennung und Minderung dieser Risiken aufgezeigt. Hierbei geht es vor allem auch um Maßnahmen zur Stärkung der Gesundheitskompetenz. Weiterhin werden an individuellen Bewältigungsund Handlungskompetenzen ansetzende Maßnahmen vorgestellt, mit denen die Widerstandsfähigkeit älterer Menschen gegenüber kritischen Lebensereignissen gestärkt werden soll.

Ziel 3: Körperliche Aktivität und

Mobilität älterer Menschen

sind gestärkt bzw. erhalten.

Die Bedeutung regelmäßiger körperlicher Aktivität für den Erhalt und die Förderung der Gesundheit ist gut belegt. ${ }^{9}$ Die Wirkungen reichen von der Verbesserung der kognitiven Funktionen über die Reduzierung von Depressionen

5 Robert Koch-Institut. Gesundheitsberichterstattung des Bundes. Heft 28 - Altersdemenz. Berlin: Robert-Koch-Institut; 2005.

6 Robert Koch-Institut. Gesundheitsberichterstattung des Bundes. Heft 51 - Depression. Berlin: RKI; 2010.

7 Robert Koch-Institut, Statistisches Bundesamt, Deutsches Zentrum für Altersfragen, editors. Beiträge zur Gesundheitsberichterstattung des Bundes. Gesundheit und Krankheit im Alter. Berlin: Robert KochInstitut; 2009; verfügbar unter: www.rki.de.

8 Bundesministerium für Familie, Senioren, Frauen und Jugend (BMFSFJ). Fünfter Bericht zur Lage der älteren Generation in der Bundesrepublik Deutschland. Potenziale des Alters in Wirtschaft und Gesellschaft. Der Beitrag der älteren Menschen zum Zusammenhalt der Generationen. Berlin: Deutscher Bundestag; 2006.; Wilson J. Volunteering. Annu. Rev. Sociol. 2000 26:215-40.].

9 (Löllgen H, Bockenhoff A, Knapp G. Physical activity and all-cause mortality: an updated meta-analysis with different intensity categories. International Journal of Sports Medicine. 2009; 30(3):213-224). 
bis hin zur Verminderung des Risikos für verschiedene Krankheiten und für einen vorzeitigen Tod. Regelmäßige körperliche Aktivitäten sind ein wichtiger Beitrag zu einer selbstständigen Lebensführung und zu Mobilität, was wiederum die Wahrnehmung gesellschaftlicher Teilhabe (siehe Ziel 1) begünstigt.

Bei der Förderung der körperlichen Aktivität und der Mobilität kommt den Kommunen eine besondere Bedeutung zu. Sie sind verantwortlich für die bewegungsförderliche Gestaltung des öffentlichen Raums und können mit einer ausreichenden Beleuchtung von Wegen und Parks, mit Ruhemöglichkeiten (Bänken), mit öffentlichen Toiletten, mit Barrierefreiheit (Gehwegabsenkungen, Aufzügen, Rampen statt Treppen) oder der Ausgestaltung des öffentlichen Personennahverkehrs viel bewirken. Sie können Maßnahmen zur Bewegungsförderung im Quartier selbst durchführen oder anstoßen, z. B. bei Volkshochschulen, bei Vereinen, insbesondere Sportvereinen oder bei Begegnungsstätten.

\section{Ziel 4: Ältere Menschen er- nähren sich ausgewogen.}

Eine vollwertige Ernährung ist bis ins hohe Alter für die Erhaltung der Leistungsfähigkeit und des körperlichen Wohlbefindens von großer Bedeutung. Neben der physiologischen Dimension haben Essen, Trinken und die Mahlzeiteneinnahme auch für ältere Menschen eine soziale, kulturelle, emotionale und psychische Bedeutung und bestimmen somit maßgeblich deren Lebensqualität. Daher sind Maßnahmen im Rahmen der Aufklärungs- und Fortbildungs- bzw. Weiterbildungsarbeit umzusetzen, die allen Beteiligten die Bedeutung einer vollwertigen Ernährung für die Erhaltung der Leistungsfähigkeit und des Wohlbefindens älterer Menschen bewusst machen. Zur Sicherstellung einer vollwertigen Ernährung für ältere Menschen, auch im eigenen Wohnumfeld, sollten entsprechende qualitätsgesicherte Angebote etabliert bzw. ausgebaut werden.

\section{Ziel 5: Die Mundgesundheit} älterer Menschen ist erhalten bzw. verbessert.

Die Mundgesundheit hat wegen der Interdependenz zwischen Mundgesund- heit und internistischen Krankheiten große Bedeutung für die allgemeine Gesundheit und wegen der Bedeutung für die Ernährung auch für die Lebensqualität. Sie wird deshalb abschließend im ersten Handlungsfeld behandelt und es werden Maßnahmen vorgeschlagen, die zum Erhalt und zur Verbesserung der Mundgesundheit älterer Menschen beitragen sollen.

Die vorgeschlagenen Maßnahmen weisen bei aller Verschiedenheit und Differenziertheit eine Reihe von Gemeinsamkeiten auf.

Für alle genannten Bereiche ist gleichermaßen wichtig, dass älteren Menschen bewusst ist, welche große Bedeutung sie für ihre Lebensqualität und ihre Gesundheit haben. Dementsprechend werden jeweils Maßnahmen der Öffentlichkeitsarbeit zur Information und zur Bewusstseinsbildung vorgeschlagen.

Für die genannten Bereiche gilt, dass jeweils auch Maßnahmen vorgeschlagen werden, die am erfolgreichsten als Intervention in der Lebenswelt oder in Umfeld oder Umwelt der älteren Menschen erfolgen. Sie liegen vielfach in der Verantwortung von Kommunen und vielfach bietet sich eine Umsetzung im jeweiligen Setting an.

Des Weiteren gilt durchweg, dass die verschiedenen sozialen Gruppen auch in verschiedener Weise angesprochen werden müssen und dass mehr als bisher auf ihre Motive, Wünsche und Bedürfnisse eingegangen wird. Dies bedeutet eine besondere Herausforderung in Bezug auf Menschen, zu denen bisher kaum Zugang gefunden worden ist, also insbesondere ältere Menschen mit geringem sozioökonomischen Status oder mit Migrationshintergrund. Wichtig ist es auch, Konzepte dafür zu entwickeln und umzusetzen, mit denen geschlechtsbezogene und soziale Ungleichheiten ausgeglichen werden.

\subsection{Medizinische, psychosoziale und pflegerische Versorgung}

Angesichts des demografischen Wandels und der Veränderung des Krankheitsspektrums stehen die Akteure und die Professionen des Gesundheitswesens vor der Aufgabe, die sektorale Aufgliederung des Gesundheitssystems zu überwinden, Umstrukturierungen vorzunehmen sowie die Verbindung präventiver, therapeutischer, rehabilitativer, pflege- rischer und beratender Dienstleistungen zu ermöglichen. Vor dem Hintergrund hat die AG folgende 3 Zielvorgaben definiert:

\section{Ziel 6: Ältere Menschen sind bei} Krankheit medizinisch, psychosozial und pflegerisch gut versorgt.

Im höheren Lebensalter steigt nicht nur die Wahrscheinlichkeit des Auftretens von Krankheiten, sondern auch die Gefahr von komplexen Krankheitsverläufen mit mehreren, zum Teil interagierenden physischen und psychischen Erkrankungen. Hierbei ist aus Sicht der AG die systematische Entwicklung, Erprobung und Implementierung von evidenzbasierten Leitlinien für die Versorgung besonders häufig auftretender Ko- und Multimorbiditäten eine wichtige Aufgabe.

Um die Gesundheitsversorgung insbesondere älterer Menschen sicherzustellen, bedarf es ferner einer ständigen Weiterentwicklung adäquater medizinischer, psychosozialer und pflegerischer Versorgungsstrukturen. Das neue GKVVersorgungsstrukturgesetz (GKV-VStG) sieht unter anderem vor, die Verzahnung der Leistungssektoren zu verbessern. Dabei ist die ambulante, vor allem die hausärztliche Versorgung von großer Bedeutung. Denn vor allem in der hausärztlichen Versorgung sind integrierte, sektorenübergreifende Konzepte für eine verbesserte Versorgung älterer Patientinnen und Patienten vonnöten. Hierfür gibt es Erfolg versprechende Ansätze, wie chronic-care-Modelle, die auf eine gesteuerte Versorgung chronisch Kranker über die Grenzen der Versorgungssektoren hinweg abzielen.

Ferner muss die (Weiter-)Entwicklung von Versorgungskonzepten gestärkt werden, die primär auf die Vermeidung einer Verschlimmerung irreversibler chronischer Erkrankungen und von Pflegebedürftigkeit ausgerichtet sind.

Ziel 7: Unterschiedliche Gesundheitsberufe arbeiten patientenorientiert und koordiniert zusammen.

Unser Gesundheitssystem bedarf eines abgestimmten und auf enge Zusammenarbeit angelegten Zusammenwirkens unterschiedlicher Gesundheitsberufe und (gesundheits-)wissenschaftlicher Disziplinen, um die Qualität der medi- 
zinischen und pflegerischen Versorgung $\mathrm{zu}$ sichern und eine verbesserte Ausschöpfung von noch vorhandenen Gesundheitspotenzialen älterer Menschen zu erreichen. Die Einführung von neuen Konzepten der Arbeitsorganisation sowie ein strukturiertes Qualitätsmanagement in der Versorgung insbesondere in stationären Einrichtungen können maßgebliche Instrumente sein, um die engere Kooperation zwischen den beteiligten Berufsgruppen zu unterstützen. Auch sind eine Stärkung geriatrischer und gerontologischer Fort- und Weiterbildung in Gesundheitsberufen sowie eine Schwerpunktlegung in Ausbildungs- und Studiengängen auf multiprofessionelle Kooperation geeignete Strategien für die Umsetzung einer patientenorientierten und koordinierten Zusammenarbeit unterschiedlicher Gesundheitsberufe.

\section{Ziel 8: Die Interessen der An-} gehörigen und der informellen Helfer(innen) von älteren Menschen sind berücksichtigt.

Die Familie, aktuell die größte und leistungsfähigste »Pflegeinstitution «, wird in Zukunft aufgrund der Zunahme allein lebender (älterer) Menschen und der $\mathrm{zu}$ erwartenden stärkeren Einbindung von (älteren) Frauen in den Arbeitsprozess einen Rückgang des familiären Pflegepotenzials erleben. ${ }^{10}$ Soll es dennoch bei dem hohen Anteil der häuslichen Pflege verbleiben, ist zukünftig ein anderes Pflegeverständnis erforderlich. Ein familienorientierter Zugriff (family bealth nursing), in welchem Angehörige bei der Betreuung und Pflege sowie in ihrer Rolle als Versorgungs- und Betreuungsinstanz unterstützt und drohenden Überlastungserscheinungen, z. B. durch Rehabilitationsmaßnahmen, vorgebeugt werden, ist sinnvoll.

Zugleich werden professionelle Unterstützungsleistungen für Pflegebedürftige und deren Angehörige an Bedeutung zunehmen. Wichtig erscheinen der Ausbau und die Vernetzung von Angeboten, die Beratung, Unterstützung und Entlastungsmöglichkeiten für pflegende Angehörige bieten. Des Weiteren müssen psychologische Barrieren (z. B. Gefühle von Scheitern/ Versagen oder Scham und Angst bei der Inanspruchnahme) abgebaut werden. Die Lebens- und Problemlagen von pflegenden Angehörigen müssen stärkere Berücksichtigung bei der Beratung und Suche nach Entlastungsmöglichkeiten erfahren.

Öffentlichkeitsarbeit und Medienpräsenz sollten dazu anregen, familienbewusste Personalpolitiken und familienfreundliche Arbeitswelten als zentrale Voraussetzungen für eine zukunftsfähige Gesellschaft anzusehen, um Arbeitnehmerinnen und Arbeitnehmer zu berücksichtigen, die zusätzlich zu ihrer Erwerbstätigkeit ihre Angehörigen im häuslichen Umfeld pflegen wollen bzw. müssen.

\section{Besondere Herausforderungen}

Im Rahmen des Wandels des Krankheitsgeschehens, den die demografische Entwicklung mit sich bringen wird, werden insbesondere Demenzerkrankungen, Chronizität und Multimorbidität sowie Pflegebedürftigkeit besondere $\mathrm{He}$ rausforderungen hervorrufen, da sie zusätzliche Anforderungen an die Versorgungs- und Unterstützungsstrukturen stellen werden. Unter diesem Handlungsfeld wurden folgende 5 Ziele formuliert.

Ziel 9: Die Gesundheit, Lebensqualität und Teilhabe von älteren Menschen mit Behinderungen sind erhalten und gestärkt.

Da die Lebensqualität und die Teilhabe von älteren Menschen mit Behinderungen wesentlich beeinträchtigt werden, beschäftigt sich das Ziel 9 mit dem Thema der Entwicklung von Umsetzungsstrategien und Maßnahmen zur Stärkung und Erhaltung der Gesundheit, Teilhabe und Lebensqualität von behinderten älteren Menschen.Laut Mikrozensus 2009 leben 9,6 Millionen Menschen mit Behinderungen in der Bundesrepublik, das sind $11,7 \%$ der Bevölkerung. ${ }^{11} \mathrm{Um}$ den älteren Menschen mit Behinderungen zu ermöglichen, ihre Lebensqualität, Gesundheit sowie ihre Teilhabe am sozialen Leben zu erhalten bzw. zu stärken, müssen vor allem präventive Strategien und Maßnahmen umgesetzt werden. ${ }^{12}$ Hierbei sollten Informationen für die Betroffenen, ihre Angehörigen und Betreuer bezüglich der Unterstützungsangebote verbessert werden, z. B. durch Kontakt- und Koordinationsstellen. Ferner sollten die Zugänglichkeit von öffentlichen Räu- men verbessert sowie räumliche und kommunikative Barrieren abgebaut werden. Auch die Entwicklung attraktiver Beschäftigungsmöglichkeiten und psychosoziale Interventionen sind geeignete Strategien zur Reduktion der verbreiteten Einsamkeitsgefühle älterer Menschen mit Behinderungen.

Ziel 10: Die psychische Gesundheit älterer Menschen ist erhalten, gestärkt bzw. wiederhergestellt.

Wie einige epidemiologische Studien zeigen, ist etwa ein Viertel der Bevölkerung über 65 Jahre von einer psychischen Erkrankung betroffen. ${ }^{13}$ Demenzielle Erkrankungen und Depressionen haben dabei eine besondere Bedeutung.

Von großer Bedeutung für die Stärkung der psychischen Gesundheit sind gesellschaftliche Angebote, die älteren Menschen Möglichkeiten zur sozialen

\section{Große Bedeutung für die} Stärkung der psychischen Gesundheit haben gesellschaftliche Angebote, die älteren Menschen Möglichkeiten zur sozialen Integration eröffnen.

Integration eröffnen. Damit die Betroffenen rechtzeitig die notwendige Unterstützung bekommen können, sind Informationen über psychische Störungen und die Möglichkeiten ihrer Behandlung wünschenswert. Dafür sind allgemeine

10 Meyer M. Pflegende Angehörige in Deutschland. Überblick über den derzeitigen Forschungsstand und zukünftige Entwicklungen. Deutsche Überarbeitung des »National Background Report of Germany« [PDF Dokument im Internet]. 2006; verfügbar unter: [zitiert am 31.03.2009].

11 Mikrozensus 2009. Statistisches Bundesamt Wiesbaden; 2011 (http://www.destatis.de/ jetspeed/portal/cms/Sites/destatis/Internet/ DE/Presse/pm/2011/05/PD11 187227. psml [zitiert am 04.01.2012].

12 Bundesministerium für Arbeit und Soziales (BMAS). Übereinkommen der Vereinten Nationen über Rechte von Menschen mit Behinderungen. Erster Staatenbericht der Bundesrepublik Deutschland; verfügbar unter: http://www.bmas.de/SharedDocs/ Downloads/DE/staatenbericht-2011.pdf? blob=publicationFile, S. 57. Stand: Juli 2011.

13 Weyerer S, Bickel H. Epidemiologie psychischer Erkrankungen im höheren Lebensalter. Grundriss Gerontologie. Band. 14. Stuttgart: Kohlhammer; 2007. 
Öffentlichkeitsarbeit, disziplinspezifische Informationsangebote über verschiedene Medien sowie niedrigschwellige Beratungsmöglichkeiten für Betroffene und ihre Angehörigen notwendig. Die Kompetenzen und der Wissensstand der Ärzte, aber auch beispielsweise der medizinischen Fachangestellten, Physiotherapeuten und Pflegekräfte in Bezug auf psychische Gesundheit und Störungen sollten verbessert werden. Dafür ist unter anderem eine bessere Vernetzung unterschiedlicher Berufsgruppen erforderlich.

Ziel 11: Demenzerkrankungen sind erkannt und Demenzerkrankte sind angemessen versorgt.

Demenzerkrankungen gehören zu den häufigsten psychischen Erkrankungen im höheren Alter. Daher sollten alle Bemühungen darauf ausgerichtet sein, die Lebensqualität Betroffener zu erhalten und das soziale Umfeld zu stabilisieren.

Eine Gesellschaft des langen Lebens muss sich auf den Umgang mit Demenz einstellen. Hierbei sind konkrete Begegnungs- und Befähigungsangebote im Lebensumfeld besonders effektiv. Zur Verfügung stehende Hilfen zur Qualifizierung von Schlüsselpersonen (z. B. Bankangestellte, Postbeamte, etc.) im alltäglichen Umgang sollten im Rahmen von Aktionen wie "Demenzfreundliche Kommune« Anwendung finden.

In der Fort- und Weiterbildung von Angehörigen der Gesundheitsberufe sollte ein stärkerer Schwerpunkt auf das Krankheitsbild Demenz gelegt werden und demenzspezifische Versorgungsstrukturen sollten weiterentwickelt werden. Die Kommunikation zwischen Ärzten und anderen Gesundheitsberufen sowie die Mitwirkung in Versorgungsnetzwerken helfen bei der Verbesserung des Umgangs mit demenzbetroffenen Familien.

Eine grundlegende Verbesserung der Versorgungssituation wird durch die Einführung eines neuen Pflegebedürftigkeitsbegriffs erwartet. Demenzerkrankte hätten dann einen besseren Zugang zu Leistungen der Pflegeversicherung.

Hilfen und Entlastungsangebote für Angehörige sollten bedarfsgerecht zur Verfügung stehen und bekannt sein (z. B. durch den Ausbau von Datenbanken).
Ziel 12: Die Versorgung multimorbider älterer Menschen ist verbessert und Folgen von Multimorbidität sind gemindert.

Aufgrund der zunehmenden Lebenserwartung haben chronisch fortschreitende Krankheitsprozesse beim älteren Menschen an Relevanz zugenommen. Sie treten häufig in Wechselwirkung mit altersbedingten physiologischen Veränderungen von Körper- und Organstrukturen auf. Hinzu kommen funktionelle gesundheitliche Einschränkungen. Zur Verbesserung der medizinischen Versorgung und zur Minderung von Krankheitsfolgen im Zusammenhang mit Multimorbidität müssen bestehende Versorgungsstandards und Versorgungsformen auf die besonderen Bedar$\mathrm{fe}$ von Patienten und Patientinnen mit Mehrfacherkrankungen abgestimmt und Lücken in der Evidenz- und Datengrundlage geschlossen werden. Zum Letztgenannten soll beispielsweise im Hinblick auf die Wirksamkeit und Sicherheit von Arzneimitteln bei älteren Menschen mit Mehrfacherkrankungen diese Zielgruppe in randomisierten und kontrollierten Studien berücksichtigt werden.

Im Rahmen der Leitlinienentwicklung bzw. Überarbeitung existierender evidenzbasierter Handlungsleitlinien zu wichtigen chronischen Erkrankungen sollten Patienten und Patientinnen mit Mehrfacherkrankungen und bestimmten Begleiterkrankungen berücksichtigt werden.

\section{Ziel 13: Pflegebedürftigkeit}

ist vorgebeugt und Pflegebe-

dürftige sind gut versorgt.

Infolge des voranschreitenden demografischen Wandels wird auch die Zahl Pflegebedürftiger künftig deutlich zunehmen. Um dieser Entwicklung zu begegnen, sind erhebliche Anstrengungen erforderlich.

Neben einer Intensivierung der Prävention von Pflegebedürftigkeit und Gesundheitsförderung in der Pflege, ist die Sicherstellung einer bedarfsangemessenen, pflegerischen Versorgung notwendig. Zwar sind mit dem PflegeVersicherungsgesetz (PflegeVG) und dem Pflege-Weiterentwicklungsgesetz in der Vergangenheit zahlreiche Anreize gesetzt worden, jedoch sind die- se noch nicht weitreichend genug. Die meisten bereits existierenden Konzepte konzentrieren sich auf die Gruppe der "jüngeren « Alten. Es sind zielgruppenspezifische Konzepte und Modelle erforderlich, die sich an Menschen im höheren Alter wenden. Diese sollten nicht nur auf Monitoring, sondern vor allem auf Aktivierung und Ressourcen- bzw. Selbstmanagementförderung abzielen und das Training körperlicher und mentaler Funktionen einbeziehen. ${ }^{14}$

Die qualitative Weiterentwicklung der ambulanten Pflege sowie die Anpassung der Angebote an alle (potenziellen) Nutzergruppen mit ihren unterschiedlichen Bedürfnissen, gehört zu den wichtigsten Aufgaben.

Notwendig ist auch die Erweiterung des Begriffs der Pflegebedürftigkeit, da der im SGB XI festgeschriebene Begriff zu eng ist, um der Bandbreite der in der Pflege vorfindbaren Bedarfslagen zu entsprechen. ${ }^{15}$

Hinweis: Das Nationale Gesund-
heitsziel "Gesund älter werden«
wird voraussichtlich im März 2012
gedruckt und veröffentlicht. Die
Publikation wird kostenfrei un-
ter www.gesundheitsziele.de zu
bestellen sein und als pdf zum
Download zur Verfügung stehen.
Download zur Verfügung stehen.

14 Walter U. Möglichkeiten der Gesundheitsförderung und Prävention im Alter. In: Kuhlmey A, Schaeffer D, editors. Alter, Gesundheit und Krankheit. Bern: Huber; 2008. p. 245-62. Schaeffer D, Büscher A, Ewers M. Ambulante pflegerische Versorgung alter Menschen. In: Kuhlmey A, Schaeffer D, editors. Alter, Gesundheit und Krankheit. Bern: Huber; 2008. p. 352-69.

15 Wingenfeld K. Stationäre pflegerische Versorgung alter Menschen. In: Kuhlmey A, Schaeffer D, editors. Alter, Gesundheit und Krankheit. Bern: Huber; 2008. p. 370-81. 